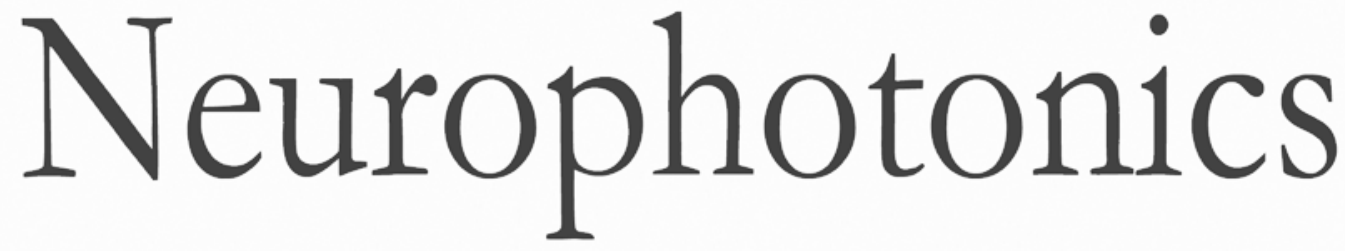

\title{
Neurovascular function recovery after focal ischemic stroke by enhancing cerebral collateral circulation via peripheral stimulation-mediated interarterial anastomosis
}

\author{
Han-Chi Pan \\ Lun-De Liao \\ Yu-Chun Lo \\ Jia-Wei Chen \\ Han-Lin Wang \\ Li Yang \\ Yao-Wen Liang \\ Po-Yu Huang \\ Ming-Hsun Yang \\ You-Yin Chen
}




\title{
Neurovascular function recovery after focal ischemic stroke by enhancing cerebral collateral circulation via peripheral stimulation-mediated interarterial anastomosis
}

\author{
Han-Chi Pan, ${ }^{a, \dagger}$ Lun-De Liao, ${ }^{\text {a, }}$ Yu-Chun Lo, ${ }^{b}$ Jia-Wei Chen,,${ }^{c}$ Han-Lin Wang, ${ }^{c}$ Li Yang, ${ }^{c}$ Yao-Wen Liang, ${ }^{d}$ \\ Po-Yu Huang, ${ }^{e}$ Ming-Hsun Yang, ${ }^{f, *}$ and You-Yin Chen ${ }^{c, *}$ \\ aNational Health Research Institutes, Institute of Biomedical Engineering and Nanomedicine, Zhunan Town, Miaoli County, Taiwan \\ ${ }^{b}$ Taipei Medical University, The PhD Program for Neural Regenerative Medicine, College of Medical Science and Technology, Taipei, Taiwan \\ 'National Yang Ming University, Department of Biomedical Engineering, Taipei, Taiwan \\ ${ }^{d}$ National Yang Ming University, Department of Life Sciences and Institute of Genome Sciences, Taipei, Taiwan \\ eNational Yang Ming University, Department of Medicine, Taipei, Taiwan \\ ${ }^{\mathrm{f}}$ Cheng Hsin General Hospital, Division of General Surgery, Department of Surgery, Taipei, Taiwan
}

\begin{abstract}
Current treatments for ischemic stroke have focused on the administration of a tissue plasminogen activator, although the associated side effects and subsequent reperfusion injury remain challenging. Peripheral electrical stimulation has shed light on therapeutic interventions for ischemia by increasing cerebral blood flow (CBF) to the target region through collateral circulation, although the mechanism remains elusive. Here, a focal photothrombotic ischemic (PTI) stroke was induced in the right hemispheric primary somatosensory forelimb cortex (S1FL) of rat brains, and the therapeutic effects of forelimb and hindlimb stimulation were characterized at the contralesional S1FL. We observed that PTI stroke rats that received forelimb stimulation exhibited significantly restored CBF of the ischemic penumbra $(1.06 \pm 0.25$ for the S1FL and $0.99 \pm 0.14$ for the primary somatosensory hindlimb cortex, respectively), electrocorticography (ECoG) delta band coherence of the intercortical S1FL $(0.93 \pm 0.04)$ at the 75 th min poststroke and an ischemic infarct $\left(7.3 \pm 0.2 \mathrm{~mm}^{3}\right)$ via collateral circulation recruitment. Importantly, anterior cerebral artery/middle cerebral artery (ACA-MCA) interarterial anastomotic regulation occurred upon forelimb stimulation and played roles in the recovery of neurovascular functions. These results indicated that receptive field-specific stimulation further restores CBF, neuronal activities, and tissue viability through the enhancement of ACA-MCA interarterial anastomosis-mediated collateral circulation and provides a feasible therapeutic intervention for stroke recovery. ๑ 2017 Society of Photo-Optical Instrumentation Engineers (SPIE) [DOI: 10.1117/1.NPh.4.3.035003]
\end{abstract}

Keywords: electrocorticography; laser speckle contrast imaging; photothrombotic ischemia; peripheral stimulation; stroke; anastomosis.

Paper 17096R received May 17, 2017; accepted for publication Sep. 12, 2017; published online Sep. 29, 2017.

\section{Introduction}

Stroke is a cardiovascular disease associated with a high risk of death, and most stroke patients suffer from irreversible neurological deficits and permanent disability due to aberrant blood supply. Stroke is a pathological condition caused by hemorrhage or occlusion of cerebral blood vessels, which ultimately leads to severe brain damage, paralysis, or death. ${ }^{1}$ Regarding ischemic stroke, clot-interrupted blood flow results in hypoxia of brain cells and the accumulation of free radicals, such as superoxide and hydroxyl radicals, and further leads to brain lesions, such as cell death and brain edema. ${ }^{2-4}$ Memezawa et al. ${ }^{5}$ suggested that blood flow reperfusion decreased the infarct volume of the ischemic penumbra of rats that underwent middle cerebral artery occlusion (MCAo). Several studies have also shown that blood reperfusion of the ischemic penumbra by a tissue plasminogen activator $^{6-8}$ or electrical stimulation ${ }^{9-12}$ rescues blood flow, neuronal functions, and infarct tissues from severe ischemic

*Address all correspondence to: Ming-Hsun Yang, E-mail: barrella.tw@yahoo com.tw; You-Yin Chen, E-mail: irradiance@so-net.net.tw

${ }^{\dagger}$ Han-Chi Pan and Lun-De Liao contributed equally to this work. damage; furthermore, the timing of poststroke treatment during the early stage of stroke is also crucial for maximizing treatment efficacy. ${ }^{11,12}$ Electrical sensory stimulation has been found to decrease stroke-induced ischemic volume and improves outcomes in rats due to neurovascular regulation upon neuronal activation, ${ }^{9,10,13,14}$ and it confers neuroprotective effects at the early stage, not the late stage, of stroke. ${ }^{10-12,15}$ In addition, previous studies have shown that collateral circulation is recruited by ischemic stroke per se or peripheral stimulation. ${ }^{9,10,12,16}$ Collateral circulation serves as a predictive outcome for hemodynamic compensation and cerebral blood flow stabilization to sustain cell metabolism and neural functions of the ischemic penumbra. ${ }^{9,10-12,16-21}$ Blood flow reversal has also been suggested as a reperfusion route for downstream blood vessels during photothrombotic ischemia (PTI). ${ }^{19}$ On the other hand, anastomotic connections between the anterior cerebral artery (ACA) and the middle cerebral artery (MCA) also develop after blood vessel occlusion ${ }^{16}$ and exhibit smaller cortical infarcts. ${ }^{22}$ However, the detailed mechanisms and functions of peripheral

2329-423X/2017/\$25.00 ๔ 2017 SPIE 
stimulation-mediated collateral circulation in ischemic stroke remain elusive.

To simultaneously examine the neurovascular functions of target brain regions, electrocorticography (ECoG) is an ideal approach that can acquire real-time neurological signals from specific large-scale brain areas, such as the primary somatosensory forelimb cortex (S1FL) and the primary somatosensory hindlimb cortex (S1HL), to evaluate cortical potential changes. In addition, cutting-edge optical imaging technologies, such as diffuse optical imaging (DOI) and laser speckle contrast imaging (LSCI), have been utilized to evaluate the hemodynamics of relative cerebral blood flow (rCBF) of stroke pathology. ${ }^{23,24}$ DOI has been shown capable of measuring the blood flow, oxygenation, and volume of brain tissues by absorption and elastic scattering of light ranging in wavelength from 650 to $950 \mathrm{~nm} \cdot{ }^{25,26}$ However, the spatial resolution of DOI is limited because the diffusion of light through brain tissues is required to obtain fine resolution $(1 \mathrm{~cm})$ of the ischemic region. ${ }^{25,27} \mathrm{LSCI}$ technology not only provides higher spatial resolution (up to $4 \mu \mathrm{m}$ ) of rCBF but also can be used to evaluate larger scan areas than DOI. Therefore, we integrated an ECoG recording with an LSCI system to estimate the full-field electrophysiology and hemodynamic changes in a PTI stroke model.

In this study, we aimed to investigate early sensory stimulation intervention and its mechanism for recruiting cerebral collateral circulation in PTI stroke rats. We combined ECoG recording and fine-resolution LSCI (ECoG-LSCI) to simultaneously measure neuronal activities and $\mathrm{CBF}$ dynamics, respectively, accompanied with peripheral stimulation intervention during the hyperacute phase of PTI stroke in rats.

\section{Materials and Methods}

\subsection{ECOG-LSCI System}

An ECoG-LSCI platform integrating ECoG recording and LSCI technology was used to simultaneously measure neuronal activity and full-field rCBF, respectively (Fig. 1). For the ECoG signal recording, three stainless steel epidural electrodes, including two electrodes placed on the S1FL and one reference electrode, were secured on the skull to acquire resting-state ECoG (RS-ECoG) using a multichannel data acquisition system (Blackrock Microsystems, Salt Lake, Utah) prior to peripheral stimulation. The ECoG signals were recorded through a headstage amplifier (gain of 2) and filtered by a 0.5 to $7500-\mathrm{Hz}$ bandpass filter. The signals were then digitized at a sampling rate of $1 \mathrm{kHz}$ using the filter settings, and a $100-\mathrm{Hz}$ low-pass filter was applied to compare changes in intercortical functional connectivity over time after PTI stroke with different peripheral stimuli. rCBF was assessed using an in-house LSCI system. Coherent light provided by a continuous-wave $(\mathrm{CW})$ laser module (660 nm; 100 mW; RM-CW04-100, Unice E-O Service Inc., Taoyuan, Taiwan) was used to illuminate the region of interest (ROI). The laser beam was expended with a planoconvex lens ( $f=75 \mathrm{~mm}$, LA1608-A, Thorlabs Inc., Newton, New Jersey) to a size of $\sim 40 \times 30 \mathrm{~mm}$ to provide even illumination of the exposed area of the cortex. The illuminated area was imaged using a 16-bit charge-coupled device (CCD) camera $(1032 \times$ 776 pixels; pixel size: $4.65 \times 4.65 \mu \mathrm{m}$, DR2-08S2M/C-EXCS, Point Grey Research Inc., Richmond, British Columbia, Canada) via an adjustable magnification lens $(0.3$ to $1 \times, f /$ 4.5 max) with a $2 \times$ extender. To satisfy the Nyquist sampling

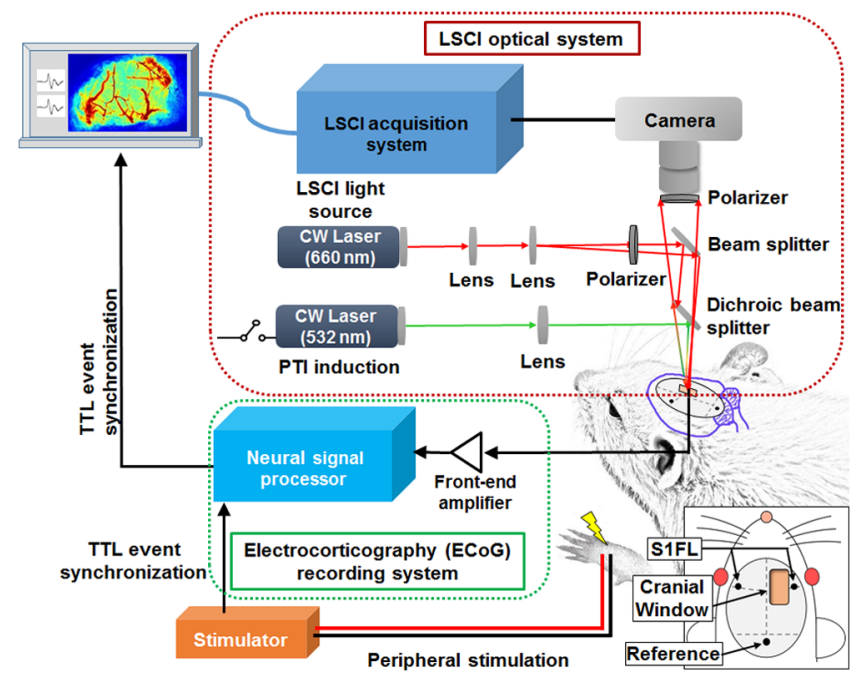

Fig. 1 Sketch of the ECoG-LSCl system and experimental setup. Schematic diagram of the ECoG-LSCl system for investigating cortical functional changes in rats. The setup included (1) ECoG recordings, (2) LSCl imaging, (3) PTI stroke in the cerebral cortex, and (4) peripheral electrical stimulation. The animal was fixed in a custom-made stereotaxic frame, and its temperature was maintained by a self-regulated heating pad. ECoG signals were recorded through two bilaterally secured epidural electrodes and one electrode (at $+2 \mathrm{~mm}$ posterior to the lambda) on the skull and were amplified via a front-end amplifier (lower right inset). For LSCI, an open-skull cranial window was made over the S1FL and $\mathrm{S} 1 \mathrm{HL}$ regions (lower right inset), and a collimated beam of a CW 660-nm laser was adjusted to evenly illuminate the cortical area of interest. The CCD camera was positioned over the exposed area with a slightly larger field of view than the craniotomy site, and the data were interconnected with a personal computer via a USB interface for further image analysis. For induction of PTI, a CW 532-nm laser was also integrated and focused on the selected distal MCA arteriole of the S1FL region of rose bengal-injected rats for $15 \mathrm{~min}$. Contralateral peripheral electrical stimulation was delivered using a DS3 stimulator.

criterion and to maximize the contrast of the imaged speckle pattern, we changed the f-stop setting of the lens to modulate the speckle size to five times the pixel pitch $(4.65 \mu \mathrm{m})$ of the CCD camera. ${ }^{28}$ To eliminate scattering, a linear polarizer was placed before the CCD image collection lens, and the working distance was $\sim 5 \mathrm{~cm}$. Laser speckle images were acquired at $30 \mathrm{fps}$, and the exposure time was fixed at $10 \mathrm{~ms}$ for in vivo experiments. The LSCI system was controlled by a custom LabVIEW program (National Instruments, Austin, Texas). A graphics processing unit (GPU) was also introduced into our LSCI data processing framework to achieve real-time, highresolution blood flow visualization on a $\mathrm{PC}$, which is a parallel computing platform and programming model invented by NVIDIA (Santa Clara, California) that allows for dramatic increases in computing performance by harnessing the power of the GPU (GeForce GTX 650 Ti, NVIDIA, Santa Clara, California). The $\mathrm{rCBF}$ of the selected ROI was acquired throughout the experiment, and changes in the rCBF of the cortical arteries and cortical veins were calculated and averaged. Nevertheless, some technical limitations of the ECoG-LSCI system still exist. For instance, bilateral ECoG recordings of the S1FL cortex through the skull-secured screw electrodes were near the imaging window of the S1FL region but could not be implanted directly in the LSCI imaging field of the S1FL to prevent interference with the optical results. 


\subsection{Animal Preparation and Surgery}

All procedures were performed in accordance with approvals from both the Institutional Animal Care and Use Committee (IACUC) of the National Health Research Institute (NHRI) and the National Yang Ming University, Taiwan. To compare the rescue effects of different peripheral stimulations on the ischemia model, a total of 45 male adult Sprague Dawley rats $(N=45)$ weighing 300 to $350 \mathrm{~g}$ were randomly allocated to three groups, including sham control, forelimb stimulation, and hindlimb stimulation groups. All animals were housed in an environment with a 12-h dark/light cycle at constant temperature and humidity and allowed free access to food and water.

The rats were anesthetized with pentobarbital $(50 \mathrm{mg} / \mathrm{kg})$ by intraperitoneal (IP) injection, and supplemental anesthesia boosters (1/3 amount of the initial dose) were given as necessary. In this study, we aimed to examine the changes in the normalized CBF in the presence of sensory stimulation; however, pentobarbital is known to partially suppress the CBF and the cerebral blood volume of rats. ${ }^{29}$ The depth of anesthesia before the surgery was confirmed by checking the response of the hindlimb withdrawal reflex. After presurgical preparation (anesthesia and hair shaving), the rats were fixed on a custom-made acrylic stereotaxic holder to reduce motion artifacts during the experiment, and the skin and muscles were removed from the skull to expose the bregma and the lambda as reference points. As shown in Fig. 1, two types of experimental setups, the ECoG recording system and the LSCI setup, were used in the experiment to simultaneously evaluate the physiological changes before and after PTI stroke induction. In the ECoG recording setup, two epidural electrodes were bilaterally fixed at the $\mathrm{S} 1 \mathrm{FL}(\mathrm{AP}=+1.0 \mathrm{~mm}, \mathrm{ML}= \pm 4.5 \mathrm{~mm})$, and a reference electrode was fixed $2 \mathrm{~mm}$ posterior to the lambda on the skull (lower right inset, Fig. 1). In the LSCI setup (lower right inset, Fig. 1), a $5 \times 2.5 \mathrm{~mm}^{2}$ cranial window was made while keeping the dura intact in the right hemisphere (diagonal coordinates of anteriorposterior $[\mathrm{AP}]:+3.5 \mathrm{~mm}$ and medial-lateral $[\mathrm{ML}]:+2 \mathrm{~mm}$; AP: $-1.5 \mathrm{~mm}$ and ML: $+4.5 \mathrm{~mm}$ to the bregma), which at the intersections of the MCA and the ACA were associated with the S1FL and the S1HL. ${ }^{15}$

\subsection{Photothrombotic Ischemic Stroke}

In this study, a branch of the MCA within the S1FL on the right hemisphere near the coordinates of $+0.25 \mathrm{~mm} \mathrm{AP}$ and $+3.5 \mathrm{~mm}$ ML to the bregma was targeted with focal PTI stroke. The target arteriole was selected based on its appearance and position within the anterior segment of the forelimb somatosensory map. ${ }^{30}$ To induce occlusion, rose bengal photosensitizer $\left(\mathrm{Na}^{+}\right.$salt, R3877; Sigma-Aldrich Corp., St. Louis, Missouri) diluted to $10 \mathrm{mg} / \mathrm{ml}$ in HEPES-buffered saline was injected through the tail vein of the anesthetized rat at $30 \mathrm{mg} / \mathrm{kg}$ body weight. Within 2 min after injection, a surface arteriole ( $80 \pm 5 \mu \mathrm{m}$ in diameter) was illuminated for $15 \mathrm{~min}$ using green-light laser (beam diameter: $0.4 \mathrm{~mm} ; 532 \mathrm{~nm}, 8.3-\mathrm{mW}$; LEDP1-G_MM400-0.48_1m_FC_M8; Doric Lenses Inc., Quebec, Canada) with a $2 \times$ optical laser lens beam expander for occlusion until a stable clot was formed. The diameter of the illumination zone for PTI was $0.8 \mathrm{~mm}$ with an average calculated light intensity of $67.5 \mathrm{~mW} / \mathrm{cm}^{2}$.

\subsection{Peripheral Electrical Stimulation}

We followed and modified the parameters of our published study. ${ }^{12}$ In brief, each rat underwent RS-ECoG and rCBF baseline measurements prior to PTI stroke. After 15-min PTI stroke, each rat sequentially underwent 10 ECoG-LSCI recording sessions with 15-min intervals. Peripheral electrical stimulation was followed by $210 \mathrm{~s}$ of ECoG-LSCI recording for each session, except for the 10th session. Representative time courses of the peripheral electrical stimulation during the 10 consecutive ECoG-LSCI recording sessions are shown in Fig. 2.

Two needle electrodes were subcutaneously inserted into a contralateral forepaw or hindpaw. The limbs were stimulated by applying trains of rectangular pulses of $200 \mu$ s duration at a repetition rate of $5 \mathrm{~Hz}$ supplied with a DS3 isolated current stimulator (Digitimer Ltd., Welwyn Garden City, Hertfordshire,

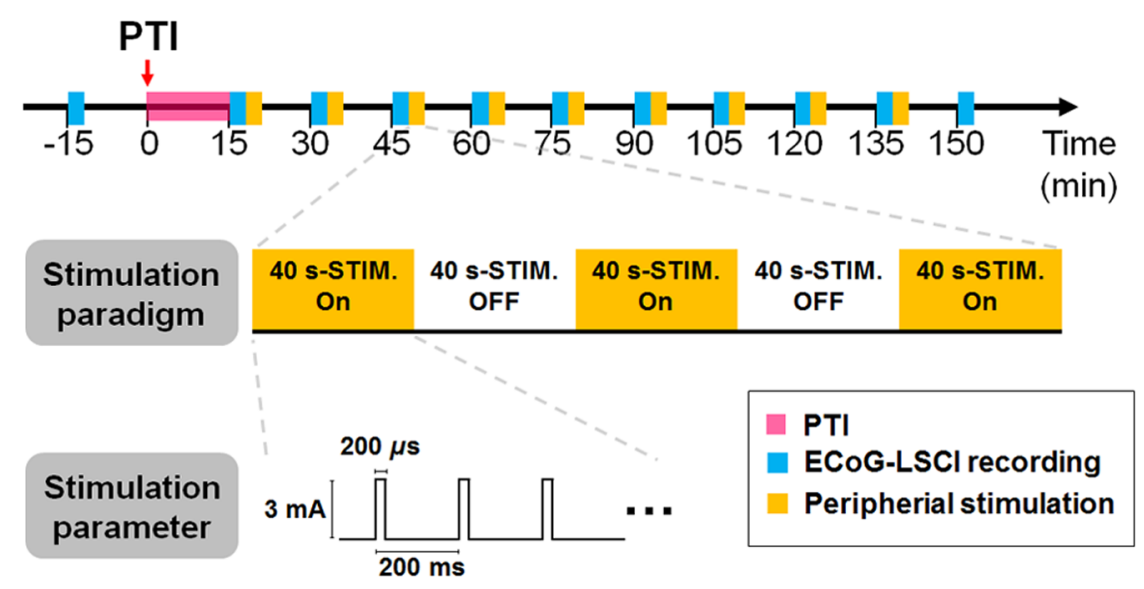

Fig. 2 The timeline of peripheral stimulation and ECoG-LSCI recording. A schematic diagram of the experimental protocol indicating the timing of PTI induction (pink box), ECoG recordings and LSCI imaging for $210 \mathrm{~s}$ (blue boxes) and sensory electrical stimulation (yellow boxes) (upper). The ECoG and LSCI signals were first recorded before PTI stroke as basal levels and further recorded every 15 min after PTI stroke (from poststroke 15 to $150 \mathrm{~min}$ ). The sensory electrical stimulation paradigm, as shown in the middle panel, consists of a stimulation train with an amplitude of $3 \mathrm{~mA}$, a pulse width of $200 \mu \mathrm{s}$, a frequency of $5 \mathrm{~Hz}$, a duration of $40 \mathrm{~s}$, and a duration interval of $40 \mathrm{~s}$ (lower). Estimation of the infarct volume using TTC staining was performed $24 \mathrm{~h}$ after PTI stroke induction. 
United Kingdom). The maximum current amplitude was $3 \mathrm{~mA}$ in all stimulations. The stimulation paradigm of a single session consisted of five blocks. Each block employed $40 \mathrm{~s}$ of stimulation, which contained 200 stimulation pulses and between 40 -s rest periods.

\subsection{Data Analysis for $L S C I$}

The ROIs for speckle flow index (SFI) images were chosen for the areas of PTI with diameters of $0.8 \mathrm{~mm}$ and positions of illumination centered at AP: $+0.25 \mathrm{~mm}$ and ML: $+3.5 \mathrm{~mm}$ to the bregma, S1FL (diagonal coordinates of AP: $+0.5 \mathrm{~mm}$ and ML: $+4.25 \mathrm{~mm}$; AP: $-1.5 \mathrm{~mm}$ and ML: $+4.5 \mathrm{~mm}$ ) and S1HL (diagonal coordinates of AP: $+0.25 \mathrm{~mm}$ and $\mathrm{ML}:+2.75 \mathrm{~mm}$; AP: $-1.5 \mathrm{~mm}$ and ML: $+2.25 \mathrm{~mm}$ ). The speckle images were computed with the algorithm based on the spatial statistics of the imaged speckle pattern running in a LabVIEW environment. ${ }^{31,32}$ The spatial laser speckle contrast, $K$, is given by

$K=\frac{\sigma}{\langle I\rangle}$,

where $\sigma$ and $\langle I\rangle$ are the standard deviation and local mean of the speckle intensity pattern in practice, respectively, measured in a window of $5 \times 5$ pixels. Fercher and Briers ${ }^{33}$ also derived an equation for $K$ based on the ratio of the correlation time $\left(\tau_{\mathrm{c}}\right)$ of the backscattered light from the sample to the camera exposure time $(T)$, as follows:

$$
K=\sqrt{\frac{\tau_{\mathrm{c}}}{2 T}\left\{1-\exp \left(\frac{-2 T}{\tau_{\mathrm{c}}}\right)\right\}} .
$$

Because faster blood flow appears more blurred in captured images than cortical tissue with slower or no flow, the degree of blurring can be quantified at each pixel as a speckle contrast value, $K$, which is correlated with relative flow dynamics. Furthermore, Eq. (2) was used to express the speckle contrast in the time-averaged speckle pattern as a function of the exposure time $(T)$ and the correlation time $\tau_{\mathrm{c}}=\frac{\lambda}{2 \pi V}$, where $V$ is the mean velocity of scatters (flow speed), and $\lambda$ is the optical wavelength of the coherent source. Equation (2) can be rewritten as

$K^{2}=\frac{\lambda}{4 \pi T V}\left\{1-\exp \left(\frac{-4 \pi T V}{\lambda}\right)\right\}$.

In this study, the optical wavelength for LSCI experiments was $660 \mathrm{~nm}$, which yielded $\frac{-4 \pi T V}{\lambda}<10^{-7}$ and implied $\exp \left(\frac{-4 \pi T V}{\lambda}\right) \rightarrow 0$. Therefore, the flow speed was considered to be represented as

$V \propto \frac{1}{T K^{2}} \equiv \mathrm{SFI}$

Note that Eq. (4) provides the rCBF via LSCI that is inversely proportional to $K^{2}$, as represented by the SFI. ${ }^{34-36}$ To produce $K^{2}$-maps, a sliding window of $5 \times 5$ pixels was applied to 10 consecutive speckle frames, which were then averaged for noise reduction.

To quantify the rCBF changes at different time points of postPTI stroke in both the ACA and MCA regions, a normalized ratio of $\mathrm{rCBF}\left(\mathrm{rCBF}_{\mathrm{N}}\right)$ from the SFI images was defined as
$\operatorname{rCBF}_{\mathrm{N}}\left(T_{n}\right)=\frac{R\left(T_{n}\right)}{R\left(T_{b}\right)}$,

where $R\left(T_{b}\right)$ is the baseline corresponding to the mean value of resting SFI fluctuations before PTI stroke, and $R\left(T_{n}\right)$ is the mean value of resting SFI fluctuations at the $n$ 'th time window post-PTI stroke.

The linear correlation of resting SFI fluctuations between the ACA and MCA regions at each frequency was evaluated by a magnitude-squared coherence function given by

$\operatorname{Coh}_{\mathrm{AM}}^{2}(f)=\frac{\left|P_{\mathrm{AM}}(f)\right|^{2}}{P_{\mathrm{AA}}(f) P_{\mathrm{MM}}(f)}$,

where A and $\mathrm{M}$ represent SFI signals of the ACA and the MCA, respectively, and $P_{\mathrm{AM}}(f)$ represents the cross power spectral density of the ACA and the MCA. $P_{\mathrm{AA}}(f)$ and $P_{\mathrm{MM}}(f)$ represent the power spectral densities of the ACA and the MCA, respectively. Note that the power spectral density was estimated by Welch's overlapped averaged periodogram method. ${ }^{37}$

In this study, the two SFI signals were coherent at the frequency band $(f)$ between 0.05 and $0.15 \mathrm{~Hz}$ where the coherence values were larger than $0.5 .^{38,39}$ Therefore, the phase of $P_{\mathrm{AM}}(f)$ at frequencies between 0.05 and $0.15 \mathrm{~Hz}$ was used to indicate the relative lag between the coherent components, which was calculated to describe the temporal relationship of SFI between the ACA and the MCA regions and is defined by

$\phi(f)=\tan ^{-1}\left[\frac{\operatorname{Im}\left[P_{\mathrm{AM}}(f)\right]}{\operatorname{Re}\left[P_{\mathrm{AM}}(f)\right]}\right]$,

where a positive phase difference $[\phi(f)]$ indicates that the ACA precedes the MCA as the blood of the ACA region is perfused through the MCA region, whereas a negative $\phi(f)$ indicates that the ACA lags the MCA as the blood of the MCA region is perfused through the ACA region. Then, the time difference can be calculated by ${ }^{40}$

$\Delta t(f)=\frac{\phi(f)}{f}$.

\subsection{Resting State Functional Connectivity Based on Bilateral ECoG Recording}

The coherence was previously utilized to analyze dynamic changes between the ECoG time series. ${ }^{41,42}$ This approach was also used in this study to investigate the correlated relationship between time courses in bilateral S1FL cortical regions. The RS-ECoG signals were measured from the time points $15 \mathrm{~min}$ before PTI stroke to $150 \mathrm{~min}$ after stroke. The signals were filtered using a Butterworth band-pass filter with low- and high-cut off frequencies of 0.5 and $50 \mathrm{~Hz}$. The temporal fluctuations of the ECoG signals from the left and right cortices were calculated for the delta $(1$ to $5 \mathrm{~Hz})$, theta $(5$ to $8 \mathrm{~Hz})$, alpha ( 8 to $15 \mathrm{~Hz}$ ), and beta $(15$ to $30 \mathrm{~Hz}$ ) frequency bands. MATLAB ${ }^{\circledR}$ was used to analyze the ECoG recordings offline.

In this study, the temporal changes in intercortical functional connectivity after PTI stroke were confirmed by serial RS-ECoG recordings. In line with previous findings, ${ }^{12,43}$ ECoG signals in the low-frequency delta band ( 1 to $5 \mathrm{~Hz}$ ) were significantly attenuated compared with before PTI stroke. Therefore, the averaged magnitude-squared coherence was used to measure 
the intercortical functional connectivity, ${ }^{44}$ which was calculated using a 210-s ECoG signal over the delta band across bilateral S1FL cortical regions. Here, the magnitude-squared coherence was calculated by

$$
\operatorname{Coh}_{\mathrm{RL}}^{2}(f)=\frac{\left|P_{\mathrm{RL}}(f)\right|^{2}}{P_{\mathrm{RR}}(f) P_{\mathrm{LL}}(f)},
$$

where R and L represent RS-ECoG signals from the right and left S1FL cortical regions, respectively. Data were analyzed offline using custom-built MATLAB ${ }^{\circledR}$ software (MATLAB R12, MathWorks Inc.). Magnitude-squared coherence (function "mscohere" in MATLAB ${ }^{\circledR}$ ) was used in this study. Magnitudesquared coherence measurement parameters include coherence frequency segment size (5000 points), Hanning window overlap $(50 \%)$ and tapering, and sampling rate $(1 \mathrm{kHz}) . P_{\mathrm{RL}}(f)$ represents the cross power spectral density between bilateral S1FL cortical regions, and $P_{\mathrm{RR}}(f)$ and $P_{\mathrm{LL}}(f)$ represent the power spectral densities of the right and left brain regions, respectively. The frequency band $(f)$ is the delta band.

A normalized ratio of $\operatorname{Coh}_{\mathrm{RL}}^{2}(f)$ is further defined by

$\mathrm{CR}_{\text {inter }}\left(T_{n}\right)=\frac{\left.\operatorname{Coh}_{\mathrm{RL}}^{2}(f)\right|_{T_{n}}}{\left.\operatorname{Coh}_{\mathrm{RL}}^{2}(f)\right|_{T_{b}}}$,

where $\left.\operatorname{Coh}_{\mathrm{RL}}^{2}(f)\right|_{T_{b}}$ is the intercortical coherence value before PTI stroke, and $\left.\mathrm{Coh}_{\mathrm{RL}}^{2}(f)\right|_{T_{n}}$ is the interhemispheric coherence value at the $n$ 'th time slot post-PTI stroke.

\subsection{Quantification of Infarct Volume}

Twenty-four hours after PTI, the rats were deeply anesthetized by $10 \%$ chloral hydrate (100 mg/kg, IP injection) and perfused with cold normal saline, and their brains were rapidly removed. We followed the experimental protocol from our published study to measure the ischemic infarct. ${ }^{12}$ Each brain was rinsed with normal saline, frozen at $-20^{\circ} \mathrm{C}$ for $10 \mathrm{~min}$, sliced into $500-\mu \mathrm{m}$ coronal sections using a rat brain slicer (Zivic Instruments, Pittsburgh, Pennsylvania), and further stained with 2\% 2,3,5-triphenyltetrazolium chloride (TTC; T8877; SigmaAldrich Corp., St. Louis, Missouri) solution at $37^{\circ} \mathrm{C}$ for $20 \mathrm{~min}$. The slices were then washed twice with normal saline and fixed with $10 \%$ formalin. After fixation, the images of the stained brain slices were examined using ImageJ (National Institutes of Health, Bethesda, Maryland), and the corrected infarct volumes in the slices were qualified by the absence of staining. The corrected infarct volume $=\{[$ total lesion volume $-($ nonischemic hemisphere volume-ischemic hemisphere volume)] / ischemic hemisphere volume $\times 100 \% .^{45-47}$

\subsection{Statistical Analysis}

All data were averaged and expressed as the mean \pm standard error (SEM). Statistical analysis was performed using PASW Statistics, version 18 (SPSS, Chicago, Illinois). To compare time series changes in the intercortical functional connectivity $\left(\mathrm{CR}_{\text {inter }}\right)$ and $\mathrm{ACA}-\mathrm{MCA}$ interarterial regulation via $\mathrm{rCBF}$ coherence and its corresponding phase delay time, the nonparametric Wilcoxon signed-rank test was conducted to test for treatment effects with time. $p<0.05$ was considered statistically significant.

Following different peripheral electrical stimuli, differences in infract volume across groups were compared using the nonparametric Kruskal-Wallis test. A probability value of $p<0.05$ was used as the criterion to determine statistical significance.

\section{Results}

\subsection{Comparison of Normalized rCBF Ratio Changes Before and After Focal PTI Stroke in the Presence of Peripheral Stimulation}

The cranial window was formed while maintaining the dura intact to study the spatial distribution of the SFI in the rat cortex, as shown in Fig. 3(a). In this study, LSCI provided two-dimensional mappings of overall blood perfusion within the brain [Fig. 3(b)]. The time course of changes in cerebral vascular morphologies was obtained before and after PTI stroke. The MCA and the ACA as well as their branches were clearly detected. Baseline SFI images were taken precisely 15 min before PTI stroke induction. Following PTI induction, blood flow decreased most prominently in the core region at the distal MCA arteriole, as shown in Fig. 3(b). Therefore, to compare the rescue effects on focal PTI stroke with different peripheral stimuli, cortical regions and subtleties of dynamic SFI responses were evaluated before, during, and after PTI. We found that the $\mathrm{rCBF}_{\mathrm{N}}$ in the sham control group was significantly decreased after PTI in a time-dependent manner compared to the basal $\mathrm{rCBF}_{\mathrm{N}}$ level before PTI onset due to clot formation [** $p<0.01$, Wilcoxon signed-rank test, $N=15$, Figs. 3(b) and 4(a)]. Interestingly, increases in $\mathrm{rCBF}$ of the penumbra areas, such as the S1FL area and the S1HL area, were shown in the first measurement (Tb15) after PTI induction but were absent in the PTI area, i.e., the ischemic core. This result may arise from the collateral circulation mediated by ischemia per se. ${ }^{16}$ Nevertheless, the $\mathrm{rCBF}_{\mathrm{N}}$ of the PTI area of forelimb-stimulated rats markedly recovered to $0.67 \pm 0.13$ compared to the other two groups from 90 min after stroke ${ }^{*} p<0.05$, Wilcoxon signed-rank test, $N=15$ ), as shown in Figs. 3(b) and 4(a). However, the $\mathrm{rCBF}_{\mathrm{N}}$ values of the PTI induction area of the sham control and hindlimb-stimulated groups exhibited continual time-dependent decreases, with $\mathrm{rCBF}_{\mathrm{N}}$ values of $0.35 \pm 0.08$ and $0.43 \pm 0.07$, respectively, from $90 \mathrm{~min}$ after stroke [Figs. 3(b) and 4(a)]. As shown in Figs. 4(b) and 4(c), the $\mathrm{rCBF}_{\mathrm{N}}$ values of the S1FL and $\mathrm{S} 1 \mathrm{HL}$ areas in the forelimb-stimulated group were significantly increased to $1.06 \pm 0.25$ and $0.99 \pm 0.14$, respectively, compared to those of the sham control group of $0.73 \pm 0.08$ and $0.70 \pm 0.07$, respectively, from $75 \mathrm{~min}$ after PTI stroke (** $p<0.01$, Wilcoxon signed-rank test, $N=15$ ). However, there was no difference in $\mathrm{rCBF}_{\mathrm{N}}$ between the sham control and hindlimb-stimulated groups. These results indicated that only forelimb stimulation restored $\mathrm{rCBF}$ in the PTI induction area, S1FL area, and S1HL area after PTI stroke.

\subsection{Comparison of ACA-MCA Interarterial Anastomotic Regulation After Focal PTI in the Presence of Peripheral Stimulation}

To characterize the interarterial anastomotic regulation of the ACA and MCA territories of the ischemic penumbra before and after the administration of peripheral stimuli, we investigated the coherence $\left[\mathrm{Coh}_{\mathrm{AM}}^{2}(f)\right]$ and phase delay $[\Delta t(f)]$ of oscillations in $\mathrm{rCBF}$ at very low frequencies $(f=0.05$ to $0.15 \mathrm{~Hz}$ ) between the ACA and MCA territories. As shown in Fig. 5, no differences in ACA-MCA $\operatorname{Coh}_{\mathrm{AM}}^{2}(f)$ and $\Delta t(f)$ over 

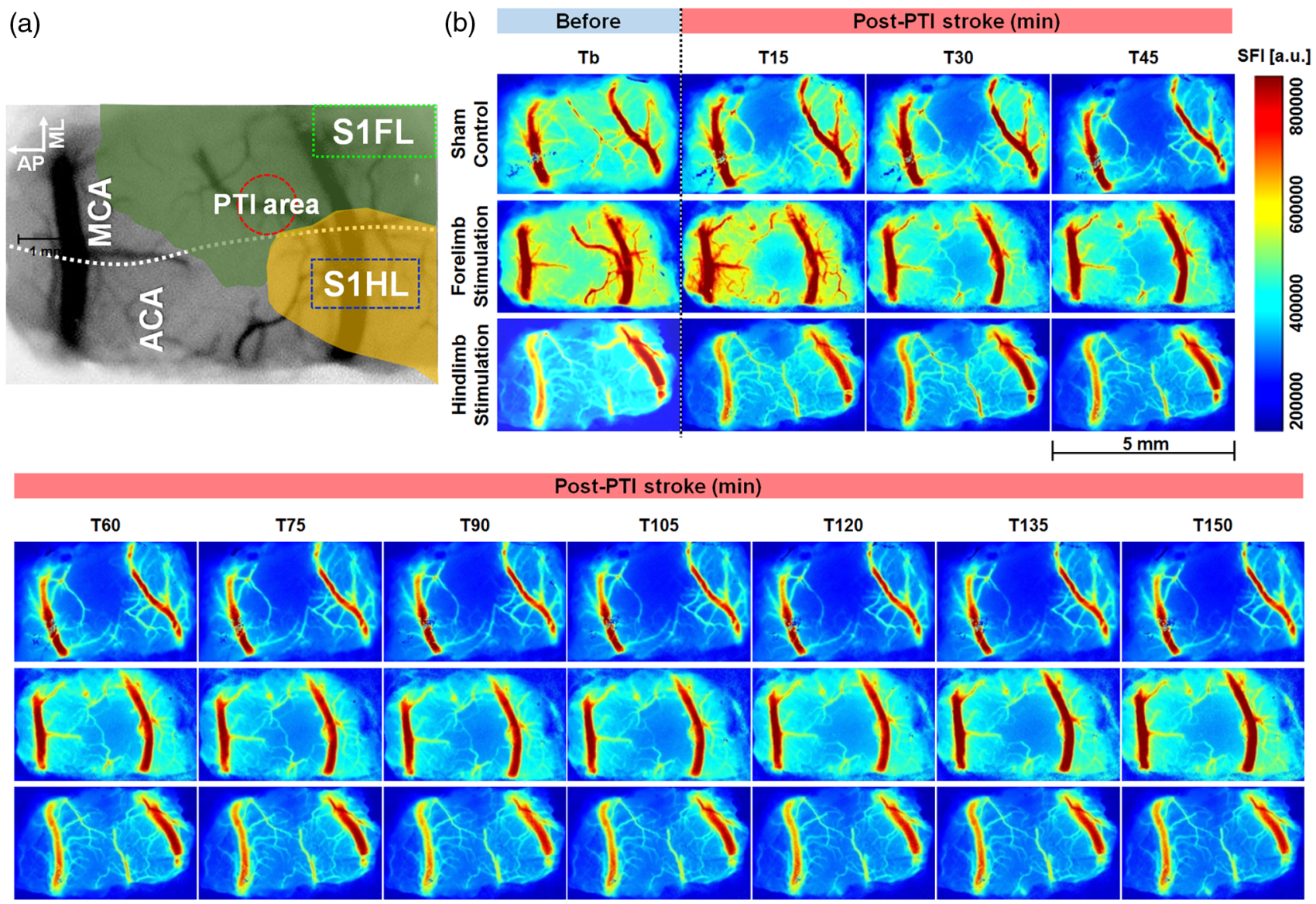

Fig. 3 The speckle contrast images for $\mathrm{rCBF}$ in the presence of peripheral stimuli. (a) Open-skull photograph of the surface of the rat brain taken prior to PTI stroke. Dorsal view of a white light image indicates the three selected ROls, including the PTI area, the S1FL, and the S1HL, over the primary somatosensory (S1) cortex. The stroke target for the S1FL cortex of PTI stroke $(1.5 \mathrm{~mm})$ and location of green laser illumination (centered at AP: $+0.25 \mathrm{~mm}$ and $\mathrm{ML}:+3.5 \mathrm{~mm}$ to bregma) is shown as a dashed red circle. The dotted green box shows an ROI of the S1FL without illumination. The dashed blue box represents an $\mathrm{ROI}$ of the S1HL. The forelimb and hindlimb receptive fields in the $\mathrm{S} 1$ cortex are represented as translucent green and yellow maps, respectively. ${ }^{48}$ The ACA-MCA collaterals are located at the interface of the distal branches of these two major supply vessels. ${ }^{49}$ The ACA-MCA anastomosis is denoted as the white dashed line. Scale bar $=1 \mathrm{~mm}$. (b) Spatiotemporal SFI evolution of the in vivo blood flow of the rat cortex showing the contralateral rCBF changes in the sham control group and upon electrical stimulation in the forelimb- and hindlimb-stimulated groups at serial time points (every $15 \mathrm{~min}$ ). Scale bar $=5 \mathrm{~mm}$. $\mathrm{Tb}$ indicates the time before PTI stroke.

(a)

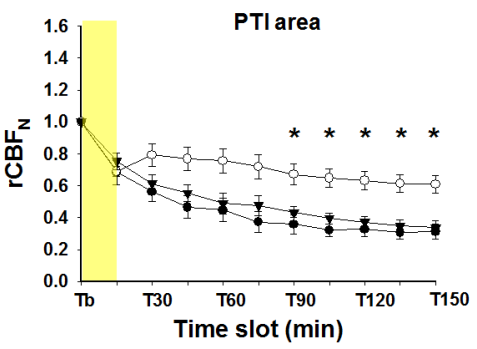

(b)

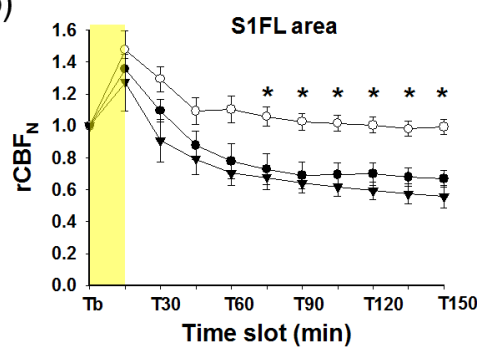

(c)

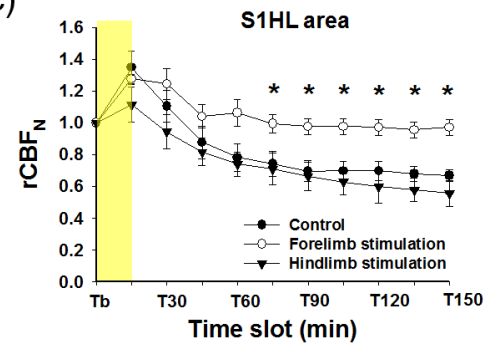

Fig. 4 The rCBF was enhanced by forelimb stimulation-induced collateral circulation. The time-series $\mathrm{rCBF}_{\mathrm{N}}$ changes in (a) the PTI area, (b) the S1FL area, and (c) the S1HL area upon different peripheral stimuli following PTI induction. The symbol "*" indicates significant differences $(p<0.05$, Wilcoxon signed-rank test) compared with controls at each time point. Data are represented as the mean \pm SEM. $N=15$ for each group. Tb indicates the time before PTI stroke.

time were observed between the sham control and hindlimbstimulated groups before and after PTI stroke. However, the ACA-MCA $\operatorname{Coh}_{\mathrm{AM}}^{2}(f)$ was significantly higher in the forelimb-stimulated group than in the other groups at 75 to $150 \mathrm{~min}$ after PTI stroke $(* * p<0.01$, Wilcoxon signed-rank test, $N=15)$. This finding revealed that the $\operatorname{Coh}_{\mathrm{AM}}^{2}(f)$ was significantly enhanced in the presence of forelimb stimulation, as it was not enhanced in the sham control or hindlimb-stimulated 
groups. Furthermore, there was an obvious increase in ACAMCA phase lead $[\Delta t(f)>0]$ in the forelimb-stimulated group compared to the other two groups at 75 to $150 \mathrm{~min}$ after PTI stroke (\#\# $p<0.01$, Wilcoxon signed-rank test, $N=5$ ), indicating that $\mathrm{rCBF}$ oscillations of the ACA territory led to oscillations of the MCA territory. Taken together, the results suggested that only the forelimb stimulation enhanced the hemodynamic coherence of the ACA and MCA areas, and the CBF oscillations of the ACA preceded the CBF oscillations of the MCA.

\subsection{Comparison of Intercortical Functional Connectivity After PTI Stroke in the Presence of Peripheral Stimulation}

To elucidate the intercortical connectivity in the presence of peripheral electrical stimulation, we recorded ECoG and analyzed the normalized intercortical delta band coherence $\left(\mathrm{CR}_{\text {inter }}\right)$. As shown in Fig. 6, a significant decrease in $\mathrm{CR}_{\text {inter }}$ was observed at 15 min after PTI stroke compared with before PTI stroke [** $p<0.01$ vs. $\mathrm{CR}_{\text {inter }}\left(T_{b}\right)$, Wilcoxon signed-rank test, $N=15]$. Similarly, the $\mathrm{CR}_{\text {inter }}$ values in the hindlimbstimulated group and the sham control group were also markedly decreased.

In the forelimb-stimulated group, $\mathrm{CR}_{\text {inter }}$ slowly recovered from 30 to $60 \mathrm{~min}$ post-PTI stroke followed by a significant improvement at $75 \mathrm{~min}$ post-PTI stroke compared with the $\mathrm{CR}_{\text {inter }}$ values of the other groups at $75 \mathrm{~min}$ post-PTI stroke $(* p<0.05$, Wilcoxon signed-rank test, $N=15) . \quad \mathrm{CR}_{\text {inter }}$ remained significantly increased up to $150 \mathrm{~min}$ post-PTI stroke, approaching $\sim 1.0$, compared with the other groups $(* * p<0.01$, Wilcoxon signed-rank test, $N=15$ ). However, no statistically significant increases in $\mathrm{CR}_{\text {inter }}$ were observed in the sham control and hindlimb-stimulated groups. Taken together, our data indicated that forelimb stimulation significantly and functionally enhanced the intercortical connectivity of the S1FL region.

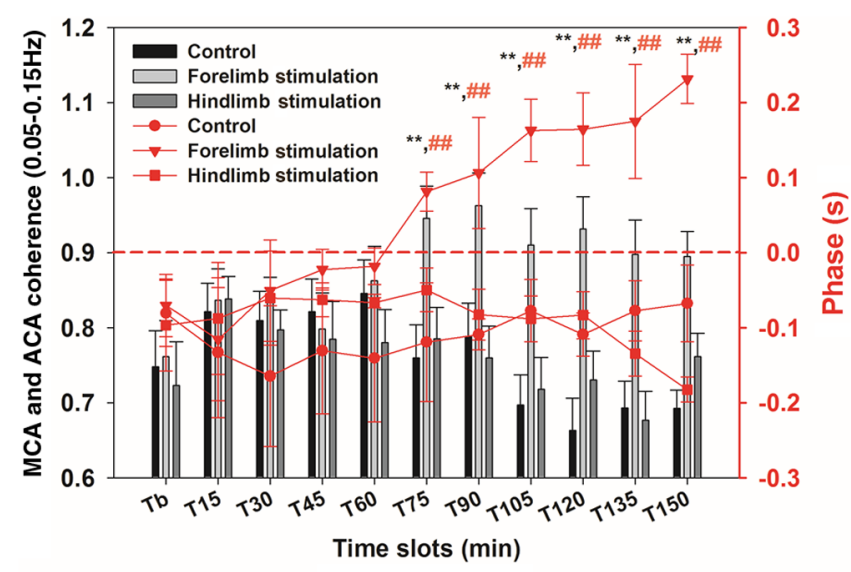

Fig. 5 Time course analysis of $\mathrm{Coh}_{\mathrm{AM}}^{2}$ and the time of phase delay between the ACA and MCA territories with different peripheral stimuli. The changes in SFI-squared coherence (bar charts) and phase delay (red solid line) functions for the sham control and the forelimb and hindlimb stimulation groups between the ACA and the MCA are plotted in 15 min intervals from the baseline recording (before PTI induction) to 150 min after PTI stroke. The red dashed line indicates the SFI time series with zero phase delay between the ACA and MCA areas. A positive phase delay indicated that SFI oscillations in the ACA led those in the MCA. The symbols "**" and "\#\#" indicate significantly different means $(p<0.01$, Wilcoxon signed-rank test) compared with controls at each time point. Data are represented as the mean \pm SEM. $N=15$ for each group. Tb indicates the time before PTI stroke.

\subsection{Comparison of Infarct Volume in the Presence of Peripheral Stimulation}

The histology of the infract volume after PTI stroke was further evaluated by TTC staining. Representative TTC-stained brain coronal sections from each group are shown in Fig. 7(a). In the sham control rats, there was apparent damage in the S1FL area of the right hemisphere, and the infarction area extended from $\sim 0.25 \mathrm{~mm}$ anterior to $0.36 \mathrm{~mm}$ posterior to the bregma to a depth of $3 \mathrm{~mm}$ from the cortex surface, which was comparable to the hindlimb-stimulated rats. In contrast, the rats that immediately received forelimb stimulation after PTI stroke exhibited less brain damage with reduced infarction $\sim 0.25 \mathrm{~mm}$ anterior to the bregma with nearly absent damage in other slices.

The statistical results revealed that PTI stroke animals that received forelimb stimulation exhibited the most significant reduction in infarct volumes by $\sim 44 \%\left(7.35 \pm 0.23 \mathrm{~mm}^{3}\right.$, $* p<0.05$, Kruskal-Wallis test, $N=15$ ) compared to the sham control rats $\left(17.05 \pm 72.71 \mathrm{~mm}^{3}\right)$ and the hindlimb-stimulated rats $\left(16.41 \pm 2.21 \mathrm{~mm}^{3}\right)$ [Fig. 7(b)].

\section{Discussion}

In this study, we combined ECoG recordings and LSCI to investigate the interhemispheric connectivity and the hemodynamics in PTI stroke rats accompanied by peripheral electrical stimulation. Contralateral forelimb stimulation improved blood flow perfusion of the S1FL region, restored functional connectivity of the brain, and reduced the infarct volume in PTI rats, whereas these improvements were absent in the hindlimb-stimulated group.

\subsection{Contralateral Forelimb Stimulation Restores rCBF in the S1FL Region of PTI Stroke Rats}

LSCI is a relatively inexpensive, fast, and noninvasive optical technique used to measure vascular blood flow by utilizing laser speckle to achieve higher spatial and temporal resolutions, ${ }^{13,24,51}$ and it has been commonly applied to estimate

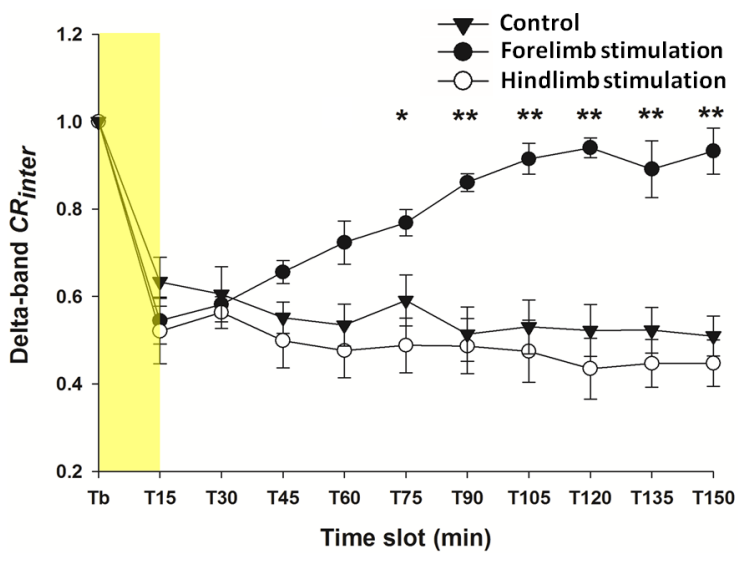

Fig. 6 Time course analysis of interhemispheric S1FL functional connectivity (RS-ECoG $\mathrm{CR}_{\text {inter }}$ ) before and after PTI stroke. Comparison of changes in the normalized delta band coherence between bilateral S1FL regions with different peripheral stimuli following PTI induction. The results indicated that coherence in the delta bands between the bilateral S1FL regions significantly recovered upon timely administration of forelimb electrical stimulation. The symbols "*” and " "**" indicate the significantly different means corresponding to $p<0.05$ and $p<0.01$ (Wilcoxon signed-rank test), respectively, compared with controls at each time point. Data are presented as the mean \pm SEM. $N=15$ for each group. Tb indicates the time before PTI stroke. 
(a)

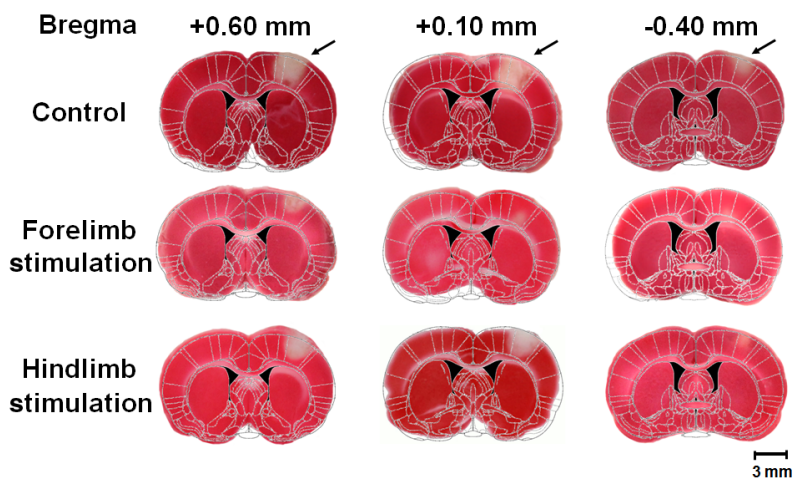

(b)

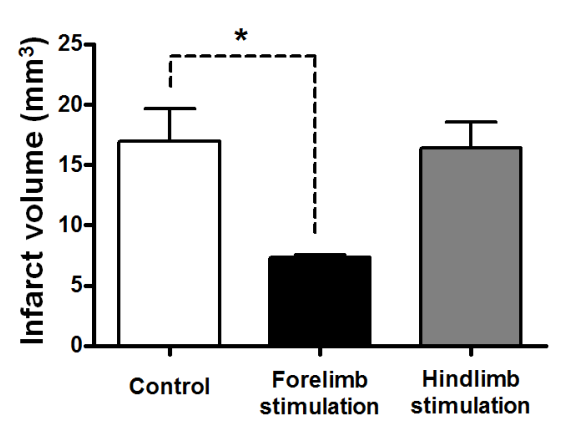

Fig. 7 Analysis and quantification of infarct volume by TTC staining of the S1FL areas according to a rat brain atlas. ${ }^{50}$ (a) Coronal sections of ischemic brain from the untreated control group (first row) and from groups that received forelimb (second row) or hindlimb (third row) stimulation after PTI stroke (the arrow indicates the infarct area). (b) Statistical analysis of infarct volume after PTI stroke. The symbol "*” indicates significantly different means ( $p<0.05$, Kruskal-Wallis test) compared with the forelimb stimulation group. Data are presented as the mean \pm SEM. $N=15$ for each group. The results showed that forelimb stimulation largely diminished the PTI stroke-induced infarct volume.

$\mathrm{CBF}$ during functional stimulation and physiological fluctuations in the cortex of rodents. ${ }^{24,52-54}$ Furthermore, LSCI has also been used to monitor cerebral hemodynamics at the scale of a single blood vessel with high spatial resolution via a cranial window $^{55}$ and to detect the development of anastomosis of collateral circulation in acute stroke rats. ${ }^{16}$ Accordingly, we measured $\mathrm{rCBF}$ of specific cortical areas, such as the S1FL and the S1HL, by LSCI and found that $\mathrm{rCBF}$ of the forelimb-stimulated group was significantly higher than rCBF of the control and hindlimb-stimulated groups after PTI stroke. These results suggest that early peripheral stimulation of target brain regions after ischemic stroke might preserve $\mathrm{CBF}$ of the infarct area via stimulated collateral circulation. These data are also consistent with a previous study that reported that functional stimulation of the sensory cortex largely reduced ischemic insult, including $\mathrm{CBF}^{56}$

\subsection{Blood Flow Pattern by LSCI Between the S1FL and S1HL Brain Regions}

Figures 4(a)-4(c) show that rCBF of the S1FL and S1HL areas immediately increased during PTI stroke onset in all groups but dropped soon after PTI stroke in the control and hindlimbstimulated groups. The rCBF of the S1FL and S1HL areas remained higher than basal levels only in the forelimb-stimulated group after PTI stroke until the end of the experiments (poststroke $150 \mathrm{~min}$ ). These results demonstrated that forelimb stimulation not only increased $\mathrm{rCBF}$ in the PTI stroke area but also in the S1FL and S1HL areas, which might be because early forelimb stimulation facilitated collateral blood flow from neighboring areas, such as the ACA and the MCA, to the PTI stroke area. Therefore, rCBF of the S1FL and S1HL areas was higher than basal levels, and therefore, rCBF increases in these three areas in the forelimb-stimulated group were higher than in the control and hindlimb-stimulated groups.

\subsection{Peripheral Stimulation-Induced Interarterial Regulation of the ACA and MCA Areas}

To investigate the mechanism of peripheral stimulation-induced collateral circulation, we monitored the coherence of blood perfusion oscillations and the phase delay time between the ACA and MCA watersheds, which might be involved in the blood perfusion change upon forelimb electrical stimulation. A previous study showed that interarterial anastomotic circulation was observed in normal rats at 29 ACA-MCA junctions per hemisphere and provides ACA collateral supply to the MCA tissue field. ${ }^{49}$ Toriumi et al. further demonstrated that the T-junctions of interarterial anastomosis of the ACA and the MCA were dynamically relocated under physiological conditions, and retrograde blood flow appeared in the MCA branch after MCAo ischemia of rodents. ${ }^{57}$ In addition, our previous study also indicated that early peripheral sensory forelimb stimulation improves the outcomes of ischemia by recruiting collateral circulation. Thus, we postulated here that the forelimb stimulationenhanced collateral circulation was associated with interarterial anastomotic regulation to reduce the ischemic damage, and the collateral perfusion may be from the ACA to the MCA. As shown in Fig. 5, the results of this study strongly suggest that only the forelimb stimulation increased the coherence of ACA and MCA hemodynamics, as the phenomenon was absent in the sham control and hindlimb stimulation groups. Moreover, the delay time of $\mathrm{rCBF}$ signals at the low frequency range $(0.05$ to $0.15 \mathrm{~Hz}$ ) of the ischemic penumbra between the ACA and MCA areas did not change in the presence of PTI at various time points in the sham control or hindlimb stimulation groups. However, it significantly increased in the forelimb-stimulated group from 75 to $105 \mathrm{~min}$ after PTI stroke, indicating that collateral perfusion of the ACA occurs prior to that of the MCA. Furthermore, the high $\mathrm{Coh}_{\mathrm{AM}}^{2}$ of the ACA and MCA areas in the forelimb-stimulated group also suggests a reliable phase relationship from the estimation. A previous study also indicated that collateral perfusion provides an alternative route for blood flow to reach ischemic tissue during stroke, ${ }^{58}$ and therefore, forelimb stimulation-enhanced collateral circulation through interarterial anastomotic regulation might play an important role in rescuing brain tissue from ischemic insults. Taken together, these results suggest that forelimb stimulation-induced collateral circulation might be highly associated with ACA-MCA interarterial anastomotic regulation, which progressed from ACA to MCA territory, after PTI stroke. 


\subsection{Peripheral Stimulation Enhances Intercortical Coherence and Rescues Brain Damage After PTI Stroke}

A previous study showed that ECoG coherence represents the functional connectivity between brain regions and is interrupted after brain injury. ${ }^{59}$ As shown in Fig. 6, the intercortical coherence of the S1FL region was largely diminished in all groups after PTI stroke, but it was enhanced only in the forelimb-stimulated group. Therefore, these results demonstrated that the functional recovery of brain damage of the S1FL region is achieved only by forelimb-sensory stimulation but not by hindlimb stimulation or in sham controls and that it might be associated with collateral circulation enhancement. The results of infarct volume after PTI stroke, as shown in Fig. 7, revealed that forelimb stimulation decreased infract volume compared to the control groups after PTI stroke. These results further corroborate the $\mathrm{CBF}$ and intercortical coherence data that only forelimb stimulation, not simulation of neighboring areas, significantly reduces the infarct volume of the S1FL region when delivered during the early stage of PTI in stroke rats and is consistent with previous studies that early peripheral stimulation exerts neuroprotective effects in stroke rats. ${ }^{10-12}$

In contrast, several studies have shown that hypoperfusion of ischemia also induces repetitive spreading depression-like depolarization, called peri-infarct depolarization (PID), in the area surrounding the ischemic core ${ }^{60-62}$ Anoxic PID is triggered by the release of potassium and excitatory amino acids, such as glutamate, from the infarct core and is propagated at a rate of $\sim 2$ to $3 \mathrm{~mm} / \mathrm{min} .{ }^{60}$ Nevertheless, von Bornstadt et al. revealed that mild tactile stimulation induces a supply-demand mismatch in the peri-infarct hot zone, known as the ischemic penumbra, and further causes stimulation-mediated PID, which worsens ischemic tissue outcome. ${ }^{63}$ However, the effects of mild tactile stimulation-triggered PIDs cannot be directly deduced to other stroke models or species. Here, based on our data, we proposed that receptive field-specific electrical stimulation enhanced interarterial anastomotic collateral circulation and rescued region-specific brain activity and tissue damage. The favorable outcome of peripheral stimulation was likely related to enhanced glucose levels by stimulation-mediated collateral perfusion blood glucose support and glycogenolysis of glial cells. ${ }^{64,65}$ However, the effects of sensory stimulation remain unclear and need to be further examined and confirmed in the future.

In summary, we measured $\mathrm{CBF}$, blood flow phase changes, and intercortical coherence by using our superresolution LSCI and ECoG recording system and examined infarct volume by TTC staining of PTI stroke rats. Early-stage electrical forelimb stimulation facilitated CBF recovery, induced changes in the blood flow phase, and enhanced collateral circulation of the infarct area and the intercortical coherence of the S1FL, which were associated with functional recovery after PTI stroke. The infarct volume was also significantly decreased in the early forelimb-stimulated group after PTI stroke. Thus, electrical stimulation administered during the early stage after PTI stroke in rats improved functional recovery and reduced infarct volume in the brain and, thus, may be a potential therapeutic strategy for clinical ischemic stroke treatment.

\section{Conclusion}

In this study, we report for the first time the use of our in-house developed ECoG-LSCI recording system to examine the functions and activities of ischemic stroke brains simultaneously with a full-field, fine spatial resolution and a high temporal resolution. PTI mediates a decrease in $\mathrm{CBF}$ and deterioration of brain activity and leads to severe brain tissue damage. Notably, peripheral stimulation of the forelimb significantly restored $\mathrm{rCBF}$ (up to $0.67 \pm 0.13,1.06 \pm 0.25$, and $0.99 \pm 0.14$ for the PTI area, S1FL, and S1HL, respectively), intercortical connectivity (up to $0.93 \pm 0.04$ ), and ischemic infarct volume $\left(7.35 \pm 70.23 \mathrm{~mm}^{3}\right)$ of PTI stroke rats compared to the sham control and hindlimb-stimulated groups. Furthermore, regionspecific peripheral stimulation induced interarterial anastomotic circulation and, therefore, may be crucial for blood reperfusion of the PTI area and for maintaining cell survival to protect the brain following an ischemic insult. Thus, peripheral stimulation enhances collateral circulation and represents a potential therapeutic strategy for the treatment of ischemic stroke.

\section{Disclosures}

No conflicts of interest, financial or otherwise, are declared by the authors.

\section{Acknowledgments}

This work was financially supported by the Ministry of Science and Technology of Taiwan under contract numbers of MOST 105-2221-E-400-006 and 105-2221-E-010-014-MY2, the Cheng Hsin General Hospital under grant number CY10516, and the National Health Research Institutes under grant number BN-106-PP-15 and BN-107-PP-15. H.C.P, L.D.L., M.H.Y., and Y.Y.C. designed the project and organized the entire study. H.C.P., M.H.Y., and Y.C.L. conceived the experiments. J.W.C., L.Y., Y.W.L., Y.W. L., and P.Y.H. implemented the hardware and software system for the experiments. H.C.P., L.D.L., M.H.Y., and Y.Y.C. conducted the experiments. H.C.P., L.D.L., Y.C.L., Y.W. L., and P.Y.H. analyzed the results. H.C.P., M.H.Y., and Y.C.L. wrote the manuscript. All authors discussed the results and reviewed the manuscript.

\section{References}

1. E. H. Lo, T. Dalkara, and M. A. Moskowitz, "Mechanisms, challenges and opportunities in stroke," Nat. Rev. Neurosci. 4(5), 399-415 (2003).

2. J. W. Schmidley, "Free radicals in central nervous system ischemia," Stroke 21(7), 1086-1090 (1990).

3. J. H. Heo, S. W. Han, and S. K. Lee, "Free radicals as triggers of brain edema formation after stroke," Free Radical Biol. Med. 39(1), 51-70 (2005).

4. N. R. Sims and H. Muyderman, "Mitochondria, oxidative metabolism and cell death in stroke," Biochim. Biophys. Acta 1802(1), 80-91 (2010).

5. H. Memezawa, M. L. Smith, and B. K. Siesjo, "Penumbral tissues salvaged by reperfusion following middle cerebral artery occlusion in rats," Stroke 23(4), 552-559 (1992).

6. T. Brott et al., "The investigational use of tPA for stroke," Ann. Emerg. Med. 17(11), 1202-1205 (1988).

7. G. J. del Zoppo, "Investigational use of tPA in acute stroke," Ann. Emerg. Med. 17(11), 1196-1201 (1988).

8. C. Fieschi et al., "Clinical and instrumental evaluation of patients with ischemic stroke within the first six hours," J. Neurol. Sci. 91(3), 311-321 (1989).

9. M. G. Burnett et al., "Electrical forepaw stimulation during reversible forebrain ischemia decreases infarct volume," Stroke 37(5), 1327-1331 (2006).

10. C. C. Lay et al., "Mild sensory stimulation completely protects the adult rodent cortex from ischemic stroke," PLoS One 5(6), e11270 (2010).

11. C. C. Lay et al., "Mild sensory stimulation reestablishes cortical function during the acute phase of ischemia," J. Neurosci. 31(32), 1149511504 (2011). 
12. L. D. Liao et al., "Rescue of cortical neurovascular functions during the hyperacute phase of ischemia by peripheral sensory stimulation," Neurobiol. Dis. 75, 53-63 (2015).

13. L. D. Liao et al., "Neurovascular coupling: in vivo optical techniques for functional brain imaging," Biomed. Eng. Online 12, 38 (2013).

14. C. Xerri and Y. Zennou-Azogui, "Early and moderate sensory stimulation exerts a protective effect on perilesion representations of somatosensory cortex after focal ischemic damage," PLoS One 9(6), e99767 (2014).

15. L. D. Liao et al., "Imaging brain hemodynamic changes during rat forepaw electrical stimulation using functional photoacoustic microscopy," NeuroImage 52(2), 562-570 (2010).

16. G. A. Armitage et al., "Laser speckle contrast imaging of collateral blood flow during acute ischemic stroke," J. Cereb. Blood Flow Metab. 30(8), 1432-1436 (2010).

17. D. S. Liebeskind, "Collateral circulation," Stroke 34(9), 2279-2284 (2003).

18. O. Y. Bang et al., "Collateral flow predicts response to endovascular therapy for acute ischemic stroke," Stroke 42(3), 693-699 (2011).

19. C. B. Schaffer et al., "Two-photon imaging of cortical surface microvessels reveals a robust redistribution in blood flow after vascular occlusion," PLoS Biol. 4(2), e22 (2006).

20. E. Cuccione et al., "Cerebral collateral circulation in experimental ischemic stroke," Exp. Transl. Stroke Med. 8, 2 (2016).

21. A. Shuaib et al., "Collateral blood vessels in acute ischaemic stroke: a potential therapeutic target," Lancet. Neurol. 10(10), 909-921 (2011).

22. H. Zhang et al., "Wide genetic variation in the native pial collateral circulation is a major determinant of variation in severity of stroke," J. Cereb. Blood Flow Metab. 30(5), 923-934 (2010).

23. J. P. Culver et al., "Diffuse optical tomography of cerebral blood flow, oxygenation, and metabolism in rat during focal ischemia," J. Cereb. Blood Flow Metab. 23(8), 911-924 (2003).

24. A. K. Dunn et al., "Dynamic imaging of cerebral blood flow using laser speckle," J. Cereb. Blood Flow Metab. 21(3), 195-201 (2001).

25. D. A. Boas, A. M. Dale, and M. A. Franceschini, "Diffuse optical imaging of brain activation: approaches to optimizing image sensitivity, resolution, and accuracy," NeuroImage 23(Suppl. 1), S275-S288 (2004).

26. A. P. Gibson, J. C. Hebden, and S. R. Arridge, "Recent advances in diffuse optical imaging," Phys. Med. Biol. 50(4), R1-R43 (2005).

27. A. Gibson and H. Dehghani, "Diffuse optical imaging," Philos. Trans. R. Soc., A 367(1900), 3055-3072 (2009).

28. S. J. Kirkpatrick, D. D. Duncan, and E. M. Wells-Gray, "Detrimental effects of speckle-pixel size matching in laser speckle contrast imaging," Opt. Lett. 33(24), 2886-2888 (2008).

29. M. M. Todd and J. Weeks, "Comparative effects of propofol, pentobarbital, and isoflurane on cerebral blood flow and blood volume," J. Neurosurg. Anesthesiol. 8(4), 296-303 (1996).

30. M. H. Mohajerani, K. Aminoltejari, and T. H. Murphy, "Targeted ministrokes produce changes in interhemispheric sensory signal processing that are indicative of disinhibition within minutes," Proc. Natl. Acad. Sci. U. S. A. 108(22), E183-E191 (2011).

31. H. Cheng et al., "Modified laser speckle imaging method with improved spatial resolution," J. Biomed. Opt. 8(3), 559-564 (2003).

32. J. C. Ramirez-San-Juan et al., "Effects of speckle/pixel size ratio on temporal and spatial speckle-contrast analysis of dynamic scattering systems: Implications for measurements of blood-flow dynamics," Biomed. Opt. Express 4(10), 1883-1889 (2013).

33. A. F. Fercher and J. D. Briers, "Flow visualization by means of singleexposure speckle photography," Opt. Commun. 37(5), 326-330 (1981).

34. I. Sigal et al., "Laser speckle contrast imaging with extended depth of field for in-vivo tissue imaging," Biomed. Opt. Express 5(1), 123-135 (2014).

35. S. M. Kazmi et al., "Expanding applications, accuracy, and interpretation of laser speckle contrast imaging of cerebral blood flow," J. Cereb. Blood Flow Metab. 35(7), 1076-1084 (2015).

36. H. Li et al., "Directly measuring absolute flow speed by frequencydomain laser speckle imaging," Opt. Express 22(17), 21079-21087 (2014).

37. P. D. Welch, "The use of fast Fourier transform for the estimation of power spectra: a method based on time averaging over short, modified periodograms," IEEE Trans. Audio Electroacoust. 15(2), 70-73 (1967).
38. L. A. Lipsitz et al., "Dynamic regulation of middle cerebral artery blood flow velocity in aging and hypertension," Stroke 31(8), 1897-1903 (2000).

39. A. P. Blaber et al., "Transfer function analysis of cerebral autoregulation dynamics in autonomic failure patients," Stroke 28(9), 1686-1692 (1997).

40. J. S. Bendat and A. G. Piersol, Engineering Applications of Correlation and Spectral Analysis, p. 315, Wiley-Interscience, New York (1980).

41. J. Hammer et al., "The role of ECoG magnitude and phase in decoding position, velocity, and acceleration during continuous motor behavior," Front. Neurosci. 7, 200 (2013).

42. C. P. Warren et al., "Synchrony in normal and focal epileptic brain: the seizure onset zone is functionally disconnected," J. Neurophysiol. 104(6), 3530-3539 (2010).

43. L. D. Liao et al., "Improving neurovascular outcomes with bilateral forepaw stimulation in a rat photothrombotic ischemic stroke model," Neurophotonics 1(1), 011007 (2014).

44. H. C. Lin et al., "Central thalamic deep-brain stimulation alters striatalthalamic connectivity in cognitive neural behavior," Front. Neural Circuits 9, 87 (2015).

45. K. Isayama, L. H. Pitts, and M. C. Nishimura, "Evaluation of 2, 3, 5triphenyltetrazolium chloride staining to delineate rat brain infarcts," Stroke 22(11), 1394-1398 (1991).

46. A. Benedek et al., "Use of TTC staining for the evaluation of tissue injury in the early phases of reperfusion after focal cerebral ischemia in rats," Brain Res. 1116(1), 159-165 (2006).

47. M. Rupadevi, S. Parasuraman, and R. Raveendran, "Protocol for middle cerebral artery occlusion by an intraluminal suture method," J. Pharmacol. Pharmacother. 2(1), 36-39 (2011).

48. T. Hjornevik et al., "Three-dimensional atlas system for mouse and rat brain imaging data," Front. Neuroinf. 1, 4 (2007).

49. P. Coyle and P. T. Jokelainen, "Dorsal cerebral arterial collaterals of the rat," Anat. Rec. 203(3), 397-404 (1982).

50. G. Paxinos and C. Watson, The Rat Brain in Stereotaxic Coordinates, Academic Press, San Diego (2007).

51. J. D. Briers, "Laser Doppler, speckle and related techniques for blood perfusion mapping and imaging," Physiol. Meas. 22(4), R35 (2001).

52. C. Ayata et al., "Laser speckle flowmetry for the study of cerebrovascular physiology in normal and ischemic mouse cortex," J. Cereb. Blood Flow Metab. 24(7), 744-755 (2004).

53. T. Durduran et al., "Spatiotemporal quantification of cerebral blood flow during functional activation in rat somatosensory cortex using laser-speckle flowmetry," J. Cereb. Blood Flow Metab. 24(5), 518-525 (2004).

54. B. Weber et al., "Optical imaging of the spatiotemporal dynamics of cerebral blood flow and oxidative metabolism in the rat barrel cortex," Eur. J. Neurosci. 20(10), 2664-2670 (2004).

55. M. B. Bouchard et al., "Ultra-fast multispectral optical imaging of cortical oxygenation, blood flow, and intracellular calcium dynamics," Opt. Express 17(18), 15670-15678 (2009).

56. C. Zhou et al., "Acute functional recovery of cerebral blood flow after forebrain ischemia in rat," J. Cereb. Blood Flow Metab. 28(7), 12751284 (2008).

57. H. Toriumi et al., "Dually supplied T-junctions in arteriolo-arteriolar anastomosis in mice: key to local hemodynamic homeostasis in normal and ischemic states?" Stroke 40(10), 3378-3383 (2009).

58. I. R. Winship et al., "Augmenting collateral blood flow during ischemic stroke via transient aortic occlusion," J. Cereb. Blood Flow Metab. 34(1), 61-71 (2014).

59. A. G. Guggisberg et al., "Mapping functional connectivity in patients with brain lesions," Ann. Neurol. 63(2), 193-203 (2008).

60. H. Nakamura et al., "Spreading depolarizations cycle around and enlarge focal ischaemic brain lesions," Brain: J. Neurol. 133(Pt 7), 1994-2006 (2010).

61. G. Mies, T. Iijima, and K. A. Hossmann, "Correlation between periinfarct DC shifts and ischaemic neuronal damage in rat," NeuroReport 4(6), 709-711 (1993).

62. K. A. Hossmann, "Periinfarct depolarizations," Cerebrovasc. Brain Metab. Rev. 8(3), 195-208 (1996).

63. D. von Bornstadt et al., "Supply-demand mismatch transients in susceptible peri-infarct hot zones explain the origins of spreading injury depolarizations," Neuron 85(5), 1117-1131 (2015). 
64. H. K. Shin et al., "Mild induced hypertension improves blood flow and oxygen metabolism in transient focal cerebral ischemia," Stroke 39(5), 1548-1555 (2008).

65. R. A. Swanson et al., "Sensory stimulation induces local cerebral glycogenolysis: demonstration by autoradiography," Neuroscience 51(2), 451-461 (1992).

Han-Chi Pan received his PhD in neuroscience from the National Yang Ming University, Taiwan, in January 2015. Currently, he is a postdoctoral researcher in the NanoNeurophotonics Lab at the Institute of Biomedical Engineering and Nanomedicine in the National Health Research Institutes, Taiwan. He is interested in neuroimaging, electrophysiology, molecular neuroscience, and brain stimulation. His long-term goal is to understand the disease mechanisms and solve the problems of neurological disorders.

Lun-De Liao received his $\mathrm{PhD}$ in electrical engineering from the National Chiao Tung University (NCTU), Taiwan, in February 2012. $\mathrm{He}$ was a postdoctoral researcher at the Brain Research Center (BRC) in NCTU. He was a research scientist in the Singapore Institute for Neurotechnology (SINAPSE) at the National University of Singapore from August 2012 to May 2014 and a senior research scientist from May 2014 to October 2015. In November 2015, he joined the Institute of Biomedical Engineering and Nanomedicine, National Health Research Institutes, Taiwan, as an assistant principal investigator and leading the NanoNeurophotonics Lab there. He has published over 65 peer-reviewed SCl journal papers, including Journal of Cerebral Blood Flow and Metabolism, Nature Scientific Reports, Proceedings of the IEEE, Neuroimage, ACS Nano and Advanced Materials journals and 12 issued patents. His research interests include neurophotonics, experimental neuroscience, and in vivo optical microscopy.

Yu-Chun Lo received his $\mathrm{PhD}$ in bioengineering from the National Taiwan University in Taiwan and is currently an assistant professor at the PhD Program for Neural Regenerative Medicine at Taipei Medical University. Her professional interests focus on behaviors and brain imaging studies of patients with mental illness and investigating communication disorders in childhood and adolescence.

Jia-Wei Chen pursues the innovation of health care and the promotion solutions using information and communication technology. $\mathrm{He}$ received complete training during $\mathrm{MS}$ degree of biomedical engineering of the National Yang Ming University and combined the knowledge from optics and photonics BS degree. He was familiar with optical simulation, image process analysis algorithm, and setting up a hardware/ software co-design system, especially for wearable device. He keeps doing the projects to improve the performance of existing medical devices for a better health.

Han-Lin Wang is a postgraduate student in the National Yang-Ming University, specializing in biomedical engineering. He works in the Neurocomputing Science and Brain-Machine Interface Lab and interests in optical measurement and development with physiological signal. He dedicates himself to figure out the mechanism of human cerebrovascular diseases.

Li Yang received his BSc degree in optics and photonics from the National Central University, Taoyuan, Taiwan, and his MSc degree in biomedical engineering from the National Yang Ming University, Taipei, Taiwan, in 2014 and 2016, respectively. Currently, he worked for National Chung-Hsing Institute of Science and Technology for 1 year, where he was involved in the advanced products design of optical electronics in modern communications.

Yao-Wen Liang is studying in the Department of Life Science, National Yang-Ming University. He is interested in the field of biomedical engineering and doing his project in Professor You-Yin, Chen's laboratory. Recently, their team has focused on developing optical system such as laser speckle contrast imaging and hopes to figure out a new method to enhance their system.

Po-Yu Huang is now a rising fifth grader majoring in medicine at the National Yang Ming University, Taiwan. He will start the clerkship at Taipei Veterans General Hospital, Taiwan. His research targets on the application of neurostimulation, including the possible relief of migraine and Parkinson's disease.

Ming-Hsun Yang received his MD degree in the medicine from the National Yang-Ming University, Taiwan, in 2004. He completed his surgery residency program from the Department of Surgery of Veteran General Hospital Taipei in 2009. Currently, he is an attending of Cheng-Hsin General Hospital. His clinical practice includes abdominal and laparoscopic surgery, hernioplasty, trauma, and emergency.

You-Yin Chen received his $\mathrm{PhD}$ in electrical engineering from the National Taiwan University, Taiwan, in 2004. Currently, he is a professor at the Department of Biomedical Engineering of the National Yang Ming University, in Taipei, Taiwan. His research interests include the development of the multifunctional neurointerface for brain-machine interface and deep brain stimulation in neurodegenerative disease, which operates at the crossroads among basic neural engineering, neurophysiology, and clinical care. 